\title{
European Journal of Medical Research reviewer acknowledgment 2014
}

Dieter Häussinger $^{1 *}$ and Johannes Waltenberger ${ }^{2}$

\section{Contributing reviewers}

The Editors of European Journal of Medical Research would like to thank all of our reviewers who have contributed to the journal in Volume 19 (2014)

\begin{abstract}
Abdel Razek Ahmed
Egypt

Addeo Raffaele

Italy

Agarwal Shiv Kumar

United States
\end{abstract}

Ahmad Umraan
United States

Akdemir Ovunc

Turkey

Aniuliene Rosita

Lithuania

Antic Darko

Serbia

Arnold Paul

United States of America

Arnold Tim

Germany

Artz Gregory J

United States of America

Asensi Victor

Spain

Assaf Naglaa

Egypt

Aytac Erman
Turkey
Banke Ingo
Germany
Barad David
United States of America
Beirer Marc
Germany
Bek Yuksel
Turkey
Berner Arne
Germany
Bevc Sebastjan
Slovenia
Bhushan Premanshu
India

Bignami Elena

Italy

Bin Song

China

Binder Thomas

Germany

Bold Richard

United States of America

\author{
Borgia Guglielmo \\ Italy \\ Bosch-Barrera Joaquim \\ Spain
}

Bostjancic Emanuela

Slovenia

Brandalize Ana Paula

Brazil

Brehm Antonio

Portugal

Breithardt Ole-A

Germany

Browning Robert

United States of America

Butera Gianfranco

Italy

Cai Lu

United States of America

Campbell Brewster

Germany

Caputo Massimo

United Kingdom

Cardoso Rhanderson

United States of America

\footnotetext{
* Correspondence: eurjmedres@biomedcentral.com

'Department of Internal Medicine, University of Düsseldorf, D-40225 Düsseldorf, Germany

${ }^{2}$ Department of Cardiovascular Medicine, University of Münster, 48149

Münster, Germany
}

(c) 2015 Häussinger and Waltenberger; licensee BioMed Central. This is an Open Access article distributed under the terms of 


\author{
Carvalho Fernanda \\ Brazil

Casini Arianna
Italy

Casso Dominguez Abel

United States of America

Castro Pablo

Chile

Cavaliere Franco

Chile

Cavlak Ugur

Turkey

Cetisli Korkmaz Nilufer

Turkey

Chen Yong

China

Chen Jiankui

China

Chihara Norio

United States of America

Christensen Robin

Denmark

Colagreco Joseph

United States of America

Cuesta Fernandez Ana

United States of America

Delhey Patrick

Germany

Deng Zhong-Liang

China

Diez Diego

Japan

Doudican Nicole

United States of America

Dragoumis Dimitrios

United Kingdom

Drenth Joost

United States of America

Economopoulos Konstantinos

United States of America

Ehrmann Stephan

France

Epiphanio Sabrina

Brazil
Fabiani Emiliano

Italy

Fenoglio Luigi

Italy

Frink Michael

Germany

Furuta Mayuko

Japan

Gaofeng Li

China

Gentile Ivan

Italy

Gill Jennifer Li

Germany

Goldberg Robert

United States of America

Gonzalez Andrew

United States of America

Greiner Jack

United States of America

Guo Lin

China

Gutiérrez-Venegas Gloria

Mexico

Han Ping

China

Hanschen Marc

Germany

Hayati Abd Rahman

Malaysia

Heggermont Ward

Belgium

Henrich Dirk

Germany

Hernández Luis

Spain

Hevezi Peter

United States of America

Hida Toyoaki

Japan

Hoff Norman-Philipp

Germany

Hu Xiaoyun

China
Hu Jian

Hong Kong

Huang Chi-Ying

Taiwan

Huang DaWei

Armenia

Huang Rongchong

China

Huang Wei

China

Hüneke Bernd

Germany

Imbeaud Sandrine

France

Irving-Rodgers Helen

Australia

Izbicki Jakob

Germany

Janowskiq John

United Kingdom

Jennings Jessica

United States of America

Jiang Tao

China

Jin Lingtao

United States of America

Juhnn Yong-Sung

South Korea

Jurisic Vladimir

Serbia

Kaiser Gernot

Germany

Kammers Kai

United States of America

Kawachi Hiroshi

Japan

Keck Tobias

Germany

Kemona Halina

Poland

Keymel Stefanie

Germany

Kim Tae Hyun

South Korea 
Kimble Roy

Australia

Kitagawa Masanobu

Japan

Kleinbongard Petra

Germany

Klostermann Cyrus

Germany

Kogut Michael

United States of America

Komm Nadja

Germany

Kosugi Shin-ichi

Japan

Kröpil Patric

Germany

Landoni Giovanni

Italy

Li Gang

China

Li Xiaojian

United States of America

Li Yuhua

China

Li Jiangtao

China

Lichte Philipp

Germany

Lin Haijiang

United States of America

Lipinski Marek

Poland

Liu Delong

United States of America

Liu Gongjun

China

Liverneaux Philippe

France

Lorand-Metze Irene

Brazil

Lorenz Kerstin

United States of America

Luk Ivi

Poland
Luo Xianghang

China

Lustig Livia

Argentina

Luton Dominique

France

Ma Lin

China

Ma Feng

China

Magalhaes Marco

United States of America

Maisch Bernhard

Germany

Major Eugene

United States of America

Makhoul Imad

Israel

Manyazewal Tsegahun

Ethiopia

Maofeng Wang

China

Marinucci Lorella

Italy

Maselli Diego

United States of America

Masroor Imrana

Pakistan

Matuschek Christiane

Germany

Mavroudis Dimitris

Greece

Mei Qibing

China

Meyer Christian

Germany

Meyer zu Hörste Gerd

Germany

Mihai Radu

United Kingdom

Miller Ashley M.

American Samoa

Miller Robert

United Kingdom
Mor Yoram

Israel

Mossad Sherif

United States of America

Motoyama Satoru

Japan

Muellenbach Ralf

Germany

Muler Kersen

Germany

Müller Christian W.

Germany

Nakabayashi Kazuhiko

Japan

Namas Rajaie

United States of America

Narod Steven

Canada

Nashan Björn

Germany

Neder Luciano

Brazil

Neuhaus Thomas

Germany

Niu Yinbo

China

Nixdorff Uwe

Germany

Noutsias Michel

Germany

Nowak-Markwitz Ewa

Poland

Noyez Luc

Netherlands

Oette Mark

Germany

Packthongsuk Kreeson

Thailand

Palomeque Julieta

Argentina

Parratt John

Australia

Pascual Angela M

Spain 
Patel Achint

United States of America

Pavenski Katerina

Canada

Pawlik Andrzej

Poland

Peiper Matthias

Germany

Perlini Stefano

Italy

Petretta Mario

Italy

Pin Richard

United States of America

Pineau Pascal

France

Plewka Danuta

Poland

Pontieri Francesco

Italy

Qin Lun-Xiu

China

Qiu Haibo

China

Ramírez-Velázquez Carlos

Mexico

Rey Johannes

Germany

Ridder Dirk De

Canada

Rodriguez Juan Carlos

Spain

Rodríguez-Ruiz David

Spain

Rohrbach Marianne

Switzerland

Rosado Balmayor Elizabeth

Germany

Rotunno Melissa

United States of America

Sakka Samir

Germany

Samra Jaswinder

Australia
Schenke-Layland Katja

Germany

Schick Martin

Germany

Seeliger Claudine

Germany

Segat Ludovica

Italy

Seow Kok-Min

Taiwan

Sethi Jigme

United States of America

Seubert Bastian

Germany

Severi Carola

Italy

Shimada Hideaki

Japan

Shulman Lee

United States of America

Sikorska Katarzyna

Poland

Song Yong

China

Sonstegard Tad S

Australia

Spears Norah

United Kingdom

Strachan Paul

United States of America

Svensson Per-Arne

Sweden

Sze Sing-Hoi

United States of America

Takeda Akihiro

Japan

Tang Zi-Hui

China

Tang Albert

China

Tappia Paramjit

Canada

Tartaglia Edoardo

Italy
Thomson Rebecca

Australia

Tillou Xavier

France

Tiwari Nidhish

United States of America

Tomaluma RC

Japan

Tong Mingjie

United States of America

Tsoutsou Pelagia

Greece

Tuomisto Anne

Finland

Van Griensven Martijn

Germany

Vancem Jeffery

United Kingdom

Vaneckova Ivana

Czech Republic

Vassiou Katerina

Greece

Verhoeven Adrie

Netherlands

Wallerman Ola

Sweden

Walocha Jerzy

Poland

Wan Song

Hong Kong

Wang Pu

China

Wang Wen

China

Wang Luhua

China

Wang Wei-Ming

China

Weig Thomas

Germany

Werdan Karl

United Kingdom

Wu Zhifeng

China 
Xu Zhen

United States of America

Xu Renshi

China

Yannarelli Gustavo

Argentina

Yao Yong-ming

China

Yarali Hakan

Turkey

Yin Leimiao

China

Yip Cheng-Har

Malaysia
Yoshida Takafumi

Japan

You Yongping

China

You Zongbing

United States of America

Yu Zhangsheng

United States of America

Yuchen Fan

China

Zeng Zihua

United States of America

Zeng Mian

China
Zhang Lin

China

Zhang Yanyun

China

Zhang Sen

China

Zhou Linuo

China

Zhu Chunfu

China

Ziaei Maryam

Australia 